\title{
Planning and Implementing Food Fortification Programs to Combat Micronutrient Malnutrition: Iron
}

\author{
Maria N. García-Casal \\ Venezuelan Institute for Scientific Research, Experimental Medicine Centre, Pathophysiology Laboratory, \\ Caracas, Venezuela \\ Email: mngarcia@ivic.gob.ve
}

Received 19 December 2013; revised 19 January 2014; accepted 26 January 2014

Copyright @ 2014 by author and Scientific Research Publishing Inc.

This work is licensed under the Creative Commons Attribution International License (CC BY). http://creativecommons.org/licenses/by/4.0/

c) (i) Open Access

\section{Abstract}

Iron deficiency anemia is the most prevalent nutritional deficiency in the world and food fortification is a cost-effective approach to combat it. This paper reviews the food fortification process with micronutrients, with special emphasis on iron and the most critical steps and common difficulties found when implementing a program. The first step is to measure the magnitude of the problem and the groups affected by iron deficiency and anemia, to determine if there is a need for fortification. Then the adequate iron compound and vehicle should be selected, to obtain a bioavailable form of iron in a frequently consumed food item without changing the taste, appearance or cooking characteristics of the final food item or complete meals containing it, to assure the acceptance by consumers. Before implementation, bioavailability studies are in order for the fortified food item and for complete meals especially typical or traditional meals, followed by field studies and pilot tests. Once implemented, the program should be monitored and evaluated continuously, and the impact on health assessed periodically to give the program the flexibility for continuation made changes or finalization when necessary. Other key elements for successful implementation of food fortification programs include the presence of a viable food industry; available channels for food marketing and distribution; a health care system to identify and monitor micronutrient malnutrition in the population; institutions for education, treatment and evaluation of the impact of the program; and continuous and effective input into the planning, implementation, monitoring and evaluation of the intervention to ensure sustainability of the intervention.

\section{Keywords}

Food Fortification, Programs, Anemia, Iron Deficiency 


\section{Introduction}

Anemia constitutes the most prevalent nutritional deficiency worldwide, especially in children and women in childbearing age. Anaemia prevalence affects $29 \%$ of non-pregnant women, $38 \%$ of pregnant women, and $43 \%$ of children worldwide [1]. The main cause of anemia in these age groups is iron deficiency. Anemia due to iron deficiency is highly prevalent in developing countries, particularly in Asia, Africa and South America, and is caused by poor dietary iron content and availability for absorption, together with increased requirements (during growth and pregnancy) and losses (especially due to intestinal parasitic infection and menstruation). Iron deficiency anemia is associated with poor physical and work performance, cognitive development and increased risk of maternal and perinatal mortality, low size or weight at birth. Given the importance of this pathology in the world, numerous countries conduct interventions to reduce anemia.

Iron deficiency is associated with considerable morbidity across the life cycle. In preschool children, iron deficiency anemia appears to be associated with potentially irreversible impairments in cognitive development and in school-aged children, iron deficiency anemia is associated with reduced school learning and educational performance as well as a controversial role on susceptibility to infections. In adults, anemia has been implicated in difficulties to perform physical work [2].

The major causes of iron deficiency include inadequate dietary iron intake due to consumption of a diet with a low iron content, or one that contains inhibitors of iron absorption [3], and increased losses of iron because of chronic blood losses, most commonly due to intestinal hookworm infection. Poor dietary intake and limited bioavailability is considered a major contributor to the global burden of iron deficiency. Populations consuming diets that chiefly comprise cereals such as maize, wheat and rice, with an inadequate intake of iron rich foods, in particular meat, but also legumes, nuts and other vegetables, are at high risk of iron deficiency [4].

In 2012, the 65th World Health Assembly approved an action plan and global targets for maternal, infant, and child nutrition, with a goal to reduce to half of the 2011 levels, the anemia prevalence in women of reproductive age by 2015 [5]. Globally the main cause of anemia is iron deficiency and is generally assumed that $50 \%$ of anemia cases are due to iron deficiency. Other micronutrient deficiencies including vitamins A and B12, folate, riboflavin and copper could also produce anemia as well as acute and chronic infections including malaria and HIV cancer and diabetes [6] [7].

Interventions against micronutrient malnutrition, considered either separately or in any combination, offer a high impact on health and micronutrient deficiencies improvement for a relatively low investment. From a variety of possible interventions (fortification, supplementation, food based approaches, change food habits, improving public health, genetically modified foods, nanotechnologies) fortification and supplementation are the least expensive interventions. Food fortification is probably the most cost-effective approach and has been proved to have an important impact on anemia prevalence in complete populations especially in the most affected age groups [8] although is not free of multiple steps and bottle necks. This paper reviews the food fortification process with iron and the most critical steps and common difficulties found when implementing a program.

\section{Food Fortification}

Fortification of staple foods and condiments with micronutrients has been used as a public health approach to reduce micronutrient deficiencies for over 100 years in many countries worldwide, showing to be both, cost-effective [9] [10] and effective although progress has been slow. Fortification of food with micronutrients is a valid technology for reducing micronutrient malnutrition as part of a food-based intervention and should be regarded as part of a broader, integrated approach to prevent micronutrient malnutrition, thereby complementing other initiatives to improve micronutrient status. Other initiatives include supplementation, change of food habits, promotion of increased consumption and/or production of food, improvement of health and sanitary conditions, biofortification, genetically modified foods and nanotechnologies.

Biofortification defined as the use of traditional plant breeding methods or genetic engineering to improve the available iron content of staple food crops, has demonstrated to increase content of zinc, carotenoids and iron in some cultivars [11] and that could be a long-term, sustainable, food-based solution for an increasing world population [12] [13]. However, increases in iron content in cereals and reserves in humans have been modest especially due to milling processes the amount of inhibitors of absorption present in flours [14] [15]. Biofortification, genetically modified foods and nanotechnologies need further development, implementation and cost-effec- 
tiveness data and evaluation to measure their impact on micronutrient deficiencies.

\section{Implementing Food Fortification Programs}

Implementing a sustainable fortification program is complicated by the fact that national programs consist of numerous components and actors. In each of these steps, there are many details to control and possible difficulties and barriers to overcome. The components include the preliminary assessment of nutrient deficiencies (in this particular case, iron), the development of fortification standards and legislation, the acquisition of equipment by industrials, the communication strategies and social marketing activities, the quality assurance and control systems and the assessment of the impact of the fortification program on health, especially on anemia and iron deficiency. Each component requires input and consensus from a number of essential organizations, such as government ministries, nutrition and health institutes, research and academic institutions, standards bureaus, industry partners, civil society and international agencies.

Table 1 shows the basic steps for implementing a fortification program. The first one is to measure the magnitude of the problem and the groups affected by iron deficiency and anemia, to determine if there is a need for fortification. Then the adequate iron compound and vehicle should be selected, to obtain the most convenient bioavailabitiy: cost ratio. The fortification procedure should not change the taste, appearance or cooking characteristics of the final food item or complete meals containing it, to assure the acceptance by consumers. Before implementation at regional or national level bioavailability studies are in order for the fortified food item and for complete meals, especially typical or traditional meals followed by field studies and pilot tests. Once implemented, the program should be monitored and evaluated continuously, and the impact on health assessed periodically to give the program the flexibility for continuation made changes or finalization when necessary.

Other key elements for successful implementation of food fortification programs include 1) the presence of a viable food industry; 2) available channels for food marketing and distribution; 3) a health care system that can help identify and monitor micronutrient malnutrition in the population; 4) institutions to provide education and treatment for deficiencies and to evaluate for instance, the impact of a food fortification program with iron on hemoglobin, ferritin and/or serum transferrin receptor concentrations and on anemia and iron deficiency prevalence; and 5) continuous and effective input into the planning, implementation, monitoring and evaluation of the intervention to help ensure sustainability of the intervention [16].

Reviewing some aspects of each step, it is important to determine the need for fortification, analyze if one of more nutrients are needed and if it is possible to add them in the same food item, and select the best fortification vehicle. Any fortification program should be based on a demonstrated need for increasing the intake of an essential nutrient in one or more population groups. It is necessary to obtain data about clinical or subclinical evidence of deficiency, estimates indicating low levels of intake of nutrients or possible deficiencies likely to develop because of changes in food habits. Ideally this information should be obtained from National health or nutrition surveys designed to answers those questions for the fortification program. If that kind of studies is not available or feasible, recent information on National food consumption data, household income and expenditure surveys (HIES), panel surveys, fortification rapid assessment tool (FRAT), FAO food balance sheets, demographic health surveys and/or industry information could be used. National policy documents, stakeholders or

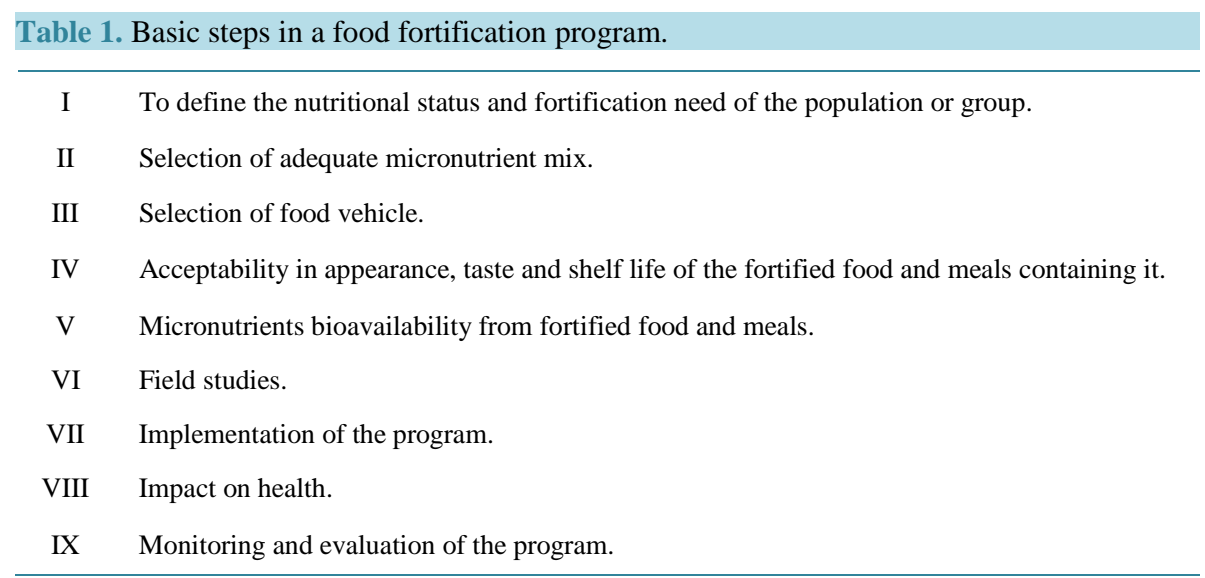


industry reports, end project evaluations and published data on sentinel, efficacy, or effectiveness studies could complement the information [17].

Fortification rapid assessment tool (FRAT) is a combination of a simplified 24 hours recall and food frequency questionnaire that could be implemented as stand-alone survey or onto an existing survey. FRAT collects representative quantitative data from household level, indicating consumption of potential food vehicles and some qualitative data on the use and availability of food vehicles, although it does not measure intake of micronutrients. Household consumption and expenditures surveys have been recently validated as tools for estimating the impact of fortified staple foods [18] [19].

To determine if mandatory or voluntary fortification is likely to be the most appropriate option it is necessary to know the significance of the public health need, being necessary to implement a mandatory program as the nutritional deficiency is more prevalent. The size and scale of the food industry sector, the political environment as well as food consumption patterns are key for both types of programs, while the level of awareness among the population about nutritional needs is more critical in case of voluntary fortification programs [17]. Knowing these factors will not only allow countries to decide if the program should be mandatory or voluntary, but also to decide if it should be a mass/universal approach or targeted to a particular region or group of population (pregnant women, children).

Once identified the nutritional needs and the magnitude of the problem, selection of the adequate iron compound or micronutrient mixture is key for a program to be successful.

Fortification with iron is technically difficult because iron reacts with food components. It is important to select an iron compound that is well absorbed and at the same time that does not change the organoleptic characteristics of the fortified item or the meals that contain the fortified food. It is also important, especially to avoid overconsumption of iron, considerations such as the distribution of iron across meals, storage under hot and humid conditions and segregation during mixing and storage [20]. In the case of flour fortification, for the choice of the best compound it is necessary to know the characteristics of the food item (for example corn or wheat flours), but also the procedure(s) involved in the production of the flour or sub products, the preparation of the bread or fortified product and also when included as part of a complete meal.

For most food vehicles the recommended iron fortificants include: ferrous sulfate, ferrous fumarate, encapsulated ferrous sulfate or fumarate. Electrolytic iron and ferric pyrophosphate have been also used, but they need to be added at twice the amount of ferrous sulfate. The co-addition of ascorbic acid is rather common in some fortification programs and is intended to enhance iron absorption. NaFeEDTA is recommended for the mass fortification of high-phytate cereal flours and for sauces with high peptide content (e.g. fish sauce, soy sauce) and ferrous bisglycinate, micronized ferric pyrophosphate and ferric ammonium citrate have been used to fortify liquid milk and dairy products, [21] [22].

Highlighting the importance of the selection of a bioavailable iron compound, a review of current wheat flour fortification programs in 78 countries conclude that the main reason for predicting little effect on iron status is the failure to specify a bioavailable iron compound. In most of these programs, millers are likely to use atomized or hydrogen-reduced iron powders because of their low cost and good sensory properties [4].

The next critical step is the selection of the food vehicle. It should be consumed by most of the population, especially the groups at greatest risk of deficiency. The consumption of the item or meals that contain it should be regular, adequate and in consistent amounts to deliver the required amounts of iron, but at the same time to avoid overconsumption and iron toxicity. The compatibility between the food item and fortification mix is also important. The mixing and distribution of the fortification mix should be a simple, inexpensive process that ensures a homogeneous distribution in the food item. The vehicle should be centrally processed in order to facilitate implementation of quality control measures and monitoring and evaluation procedures. In general, but especially in the case of fat containing foods or whole flours, the fortified item should be consumed relatively soon after production and purchasing to maintain its organoleptic characteristics and nutritional content.

Cereal flours (wheat and maize) are currently the most common vehicles for iron fortification to reach the general population. Rice is an important staple food for more than half of the population of the world, most of them living in underdeveloped countries with high incidences of micronutrients deficiencies. Polished rice has a low iron content and the efforts to fortify rice have been intense for more than 20 years. Several techniques have been developed and reported including dusting, coating, hot extrusion and cold extrusion and the impact of rice fortification programs with iron, has been positive but susceptible to improvements [23] [24].

There have been interesting efforts for fortification of condiments: curry powder, fish sauce, soy sauce, sugar 
and salt, especially with NaFeEDTA [25]-[33]. Some advantages of fortifying condiments include that they are part of the daily diet in most countries, widely consumed, reach vulnerable populations, can be added to multiple foods and can be combined with fortified staple foods [34]. However, those characteristics also made them prone to overconsumption and at risk of excessive iron intake.

The inclusion of more than one micronutrient in the same food item could be desirable and practical. In addition to treat or prevent iron deficiency and anemia, fortification could be a good opportunity to control vitamin A, iodine or other micronutrient deficiencies that may coexist in many populations. The micronutrients and the amounts to be added will primarily depend on the prevalence of deficiency (ies) and the magnitude of the problem. The decision should not be based on the number of nutrients that a particular food item will "accept", but in the actual needs of the population. Then, considerations about biochemical characteristics of the micronutrient(s) or interactions between them or with the food item should be analyzed. This includes active form of compounds, $\mathrm{pH}$, interactions, changes in organoleptic properties of the final product, etc.

In relation with the food item to be fortified the nutrient composition, as well as the natural content of inhibitors (phytates, polyphenols, calcium) or enhancers of iron absorption (vitamin C, betacarotene) should be determined as well as the possible variations during industrial processing or household preparation, either losses (i.e. vitamin C) or increases (i.e. calcium or niacin during nixtamalization).

The interrelation in micronutrients metabolism and utilization is well known. The deficiency in one micronutrient can impair the utilization of another, as well as improving nutritional status of one micronutrient may have wider benefits on health. For example, vitamin A and iron metabolisms are affected by the deficiency of the other and goitre is more resistant to improvement by iodine supplementation in the presence of iron deficiency [35]-[38]. Deficiencies of vitamin B12, folate, riboflavin and several other micronutrients can also contribute to anemia, while vitamin C and carotenoids improve iron absorption [17] [39].

Before implementation of a fortification program, either universal or targeted, there are several test and studies that need to be done. First the acceptability in appearance, taste and shelf life of the fortified food and meals containing it should be performed at pilot scale to assure acceptance of the product or meals by consumers. It is desirable to test the fortified product to the limit, in the sense of preparation, temperature and storage conditions. For example, controlled conditions for arepa preparation from fortified precooked corn flour in Venezuela, showed no changes in organoleptic characteristics for most of the regions and conditions of preparation tested. However, in one region of the country where arepas were prepared the day before consumption and with water with high-mineral content, the arepas turned green and were rejected by consumers. Second in vitro and in vivo tests of iron or micronutrient bioavailability from fortified food and meals are required, followed by small scale field studies, to determine that the fortified food is well accepted and tolerated, that iron is in the food and also, and more importantly that it is absorbed and utilizable.

The program is then implemented but this is not the end of the process, since it is necessary to measure the impact of the program and monitor and evaluate it. To evaluate the impact of the program on anemia prevalence, a baseline prevalence of anemia and iron deficiency is required. This information is probably available from the initial surveys and data sources used to justify and implement the fortification program. If the information is not available, prevalence of deficiency (ies) in a representative sampling should be determined before implementing the program to be able to measure impact once the program is started.

Despite its low sensibility and sensitivity, the biochemical parameter needed to measure the prevalence of anemia is the concentration of hemoglobin. Other indicators that could be used besides hemoglobin are serum ferritin concentration, transferrin receptor or zinc protoporphyrin [40] [41]. For example, it has been indicated that serum ferritin assesses storage iron, while serum iron and percentage of transferrin saturation reflect iron supply to tissues. Serum transferrin receptors, erythrocyte ferritin and red cell zinc protoporphyrin are indicators of iron supply to bone marrow. There are other indicators related to the consequences of anemia and iron deficiency that could also be evaluated such as annual productivity losses (Fe), physical and cognitive function, maternal morbidity and morbility.

If the program was implemented with other approaches to improve nutrition or public health, they should be addresses and measured. Those measures include the control of conditions that favor iron or other micronutrient deficiencies i.e., infections (malaria especially), hookworm infections and absorption problems as well as improvement of sanitation and living conditions and the promotion of measures such as delayed cord clamping and nutrition education.

Besides from a well-planned and structured fortification program there are some social considerations to take 
into account to improve the coverage of the program in order to reach people with limited access to fortified food. It is important to explore the barriers (i.e. living from own production, obtaining the flour from small mills not fortifying or for economic reasons) that could be preventing equal access to fortified products among the most vulnerable and implement social protection initiatives to provide fortified products.

There are some key additional issues that could help to accelerate fortification programs such as having legislation about fortification (not only when mandatory or universal, but also for voluntary fortification initiatives), the commitment of industrials, the acquisition of factory equipment and training of personnel, building laboratory capacities and obtaining the adequate, certified micronutrient premix.

Difficulties and challenges will arise and program planners must react by redesigning the complete program or by modifying how a program component is implemented, whether if they are internal factors, such as inadequate compliance in quality assurance or monitor systems, or external factors, such as fluctuations in fortification premixes, changes in consumption patterns, market shifts or the arrival of unfortified cheaper products [17].

Once the program is implemented, monitoring and evaluation is in order to provide ongoing information on the progress of implementation and to measure the health impact among intended beneficiaries. Monitoring is the continuous, ongoing collection, review, analysis, and use of information and outcomes, to assess how the program is performing against predefined criteria. The objectives when monitoring a fortification program are to ensure that fortified foods meet nutrient content and safety standards, to study access, utilization and coverage of fortified foods by consumers and to effectively manage and sustain the fortification program to eliminate vitamin and mineral deficiencies. Evaluation is the objective assessment of a program that covers its need, design, implementation, effectiveness, efficiency and sustainability. In evaluating a program the aims are to analyze why intended impacts were or not achieved, to explore unintended results and to inform practice, decisionmaking and policy [42] [43].

Designing a monitoring and evaluation framework for an iron fortification program is not a simple task and there is not a preconceived model. It needs to respond to each country reality in terms for example of aspects food industry, population consumption habits or geographical location. It has to be periodical and able to respond to the variations of the program and to political or economic changes in a country or region.

Reviewing literature about country experiences and meeting reports, besides from determining the magnitude of the iron deficiency and anemia problem, searching for the right biochemical marker to measure impact and the need to select bioavailable iron compounds, there is also a coincidence about the need to reinforce the areas of regulatory monitoring, household/individual monitoring and evaluation, and communication and social marketing, as areas usually neglected or not prioritized.

\section{Concluding Remarks}

Food fortification programs are the most cost-effective approach to combat iron deficiency and anemia in combination with other initiatives that include control of diseases, improvement of sanitation and quality of life, supplementation, food fortification, change of food habits, improvement of micronutrient content of staples crops through biofortification, genetically modified foods, nanotechnologies and nutritional education. These strategies are complementary, with their relative importance depending on local conditions and the specific local needs.

Although an effective approach, fortification with multiple micronutrients should be based on analysis of population needs, proper food vehicle and fortification mixtures. Making a food item a "medicine or supplement" is not desirable and for practical and coverage reasons it is also interesting to consider fortifying more than one food item.

Despite all the efforts and improvement in fortification programs worldwide, they have had a low impact in the reduction of anemia in some populations. The reasons are related to low consumption of the food item, the iron compound used, weak or nonexistent enforcement of regulations and quality control systems and poor manufacturing techniques and standards.

Fortification programs MUST be susceptible to constant evaluation and CHANGED (based in evidence), in order to make decisions to adjust, maintain, expand or terminate the programs, as needed. Even in presence of unstable political, social and economic conditions, countries must take the next steps to secure the continuity or sustainability of their programs.

National fortification programs take time to establish and their long-term success require active and continuous improvements and a rapid capacity for response and change, based in data from constant monitoring and 
evaluation in order to remain effective and relevant.

\section{References}

[1] Stevens, G.A., Finucane, M.M., De-Regil, L.M., Paciorek, C.J., Flaxman, S.R., Branca, F., Peña-Rosas, J.P, Bhutta, Z.A. and Ezzati, M. (2013) Global, Regional, and National Trends in Haemoglobin Concentration and Prevalence of total and Severe Anaemia in Children and Pregnant and Non-Pregnant Women for 1995-2011: A Systematic Analysis of Population-Representative Data. The Lancet Global Health, 1, e16-e25. http://dx.doi.org/10.1016/S2214-109X(13)70001-9

[2] Beard, J. (2007) Recent Evidence from Human and Animal Studies Regarding Iron Status and Infant Development. Journal of Nutrition, 137, 524S-30S.

[3] Nair, K.M., Iyengar, V. (2009) Iron Content, Bioavailability \& Factors Affecting Iron Status of Indians. Indian Journal of Medical Research, 130, 634-645.

[4] Hurrell, R., Ranum, P., de Pee, S., Biebinger, R., Hulthen, L. and Johnson, Q. (2010) Revised Recommendations for Iron Fortification of Wheat Flour and an Evaluation of the Expected Impact of Current National Wheat Flour Fortification Programs. Food and Nutrition Bulletin, 31, S7-S21.

[5] World Health Organization (2012) Comprehensive Implementation Plan on Maternal, Infant and Young Child Nutrition. Sixty-Fifth World Health Assembly, A65/11 - 26 April 2012 and A65/11 Corr.1 - 11 May 2012.

[6] Gangat, N. and Wolanskyj, A. (2013) Anemia of Chronic Disease. Seminars in Hematology, 50, 232-238. http://dx.doi.org/10.1053/j.seminhematol.2013.06.006

[7] Lawen, A. and Lane, D. (2013) Mammalian Iron Homeostasis in Health and Disease: Uptake, Storage, Transport, and Molecular Mechanisms of Action. Antioxidants \& Redox Signaling, 18, 2473-2507. http://dx.doi.org/10.1089/ars.2011.4271

[8] Gera, T., Singh, H. and Boy E. (2012) Effect of Iron-Fortified Foods on Hematologic and Biological Outcomes: Systematic Review of Randomized Controlled Trials. American Journal of Clinical Nutrition, 96, 309-324. http://dx.doi.org/10.3945/ajcn.111.031500

[9] Horton, S. (2006) The Economics of Food Fortification. Journal of Nutrition, 136, 1068-1071.

[10] Horton, S. and Ross, J. (2003) The Economics of Iron Deficiency. Food Policy, 28, 51-75. http://dx.doi.org/10.1016/S0306-9192(02)00070-2

[11] Hotz, C. and McClafferty, B. (2007) From Harvest to Health: Challenges for Developing Biofortified Staple Foods and Determining Their Impact on Micronutrient Status. Food and Nutrition Bulletin, 28, S271-S279.

[12] Nestel, P., Bouis, H., Meenakshi, J. and Pfeiffer, W. (2006) Biofortification of Staple Food Crops. Journal of Nutrition, 136, 1064-1067.

[13] Mayer, J., Pfeiffer, W. and Beyer, P. (2008) Biofortified Crops to Alleviate Micronutrient Malnutrition. Current Opinion in Plant Biology, 11, 166-170. http://dx.doi.org/10.1016/j.pbi.2008.01.007

[14] Donangelo, C., Woodhouse, L., King, S., Toffolo, G., Shames, D., Viteri, F., Cheng, Z., Welch, R. and King, J. (2003) Iron and Zinc Absorption from Two Bean (Phaseolus vulgaris L.) Genotypes in Young Women. Journal of Agriculture and Food Chemistry, 51, 5137-5143. http://dx.doi.org/10.1021/jf030151w

[15] Haas, J., Beard, J., Murray-Kolb, L., DelMundo, A., Felix, A. and Gregorio, G. (2005) Iron-Biofortified Rice Improves the Iron Stores of Nonanemic Filipino Women. Journal of Nutrition, 135, 2823-2830.

[16] Howson, C.P., Kennedy, E.T. and Horwitz, A., Committee on Micronutrient Deficiencies, Institute of Medicine (1998) Prevention of Micronutrient Deficiencies: Tools for Policymakers and Public Health Workers. National Academy Press, Washington DC, 1-224.

[17] Allen, L., de Benoist, B., Dary, O. and Hurrell, R., World Health Organization and Food and Agriculture Organization of the United Nations (WHO/FAO) (2006) Guidelines on Food Fortification with Micronutrients. 1-341.

[18] Dary, O. and Jariseta, Z. (2012) Validation of Dietary Applications of Household Consumption and Expenditures Surveys (HCES) against a 24-Hour Recall Method in Uganda. Food and Nutrition Bulletin, 33, S190-S198.

[19] Imhoff-Kunsch, B., Flores, R., Dary, O. and Martorell, R. (2012) Methods of Using Household Consumption and Expenditures Survey (HCES) Data to Estimate the Potential Nutritional Impact of Fortified Staple Foods. Food and Nutrition Bulletin, 33, S185-S189.

[20] Mannar, V. and Boy, E. (2002) Iron Fortification: Country Level Experiences and Lessons Learned. Journal of Nutrition, 132, 856S-858S.

[21] Dary, O. (2002) Staple Food Fortification with Iron: A Multifactorial Decision. Nutrition Reviews, 60, S34-S41. http://dx.doi.org/10.1301/002966402320285083 
[22] World Health Organization, Food and Agriculture Organization of the United Nations, United Nations Children’s Fund, Global Alliance for Improved Nutrition, The Micronutrient Initiative and Flour Fortification Initiative (2009) Recommendations on Wheat and Maize Flour Fortification. Meeting Report: Interim Consensus Statement. World Health Organization, Geneva. Accessed September 2013.

http://www.who.int/nutrition/publications/micronutrients/wheat_maize_fort.pdf

[23] Hotz, C., Porcayo, M., Onofre, G., García-Guerra, A., Elliott, T., Jankowski, S. and Greiner, T. (2008) Efficacy of Iron-Fortified Ultra Rice in Improving the Iron Status of Women in Mexico. Food and Nutrition Bulletin, 29, 140-149.

[24] Moretti, D., Lee, T., Zimmermann, M., Nuessli, J. and Hurrell, R. (2005) Development and Evaluation of Iron-Fortified Extruded Rice Grains. Journal of Food Science, 70, S330-S336. http://dx.doi.org/10.1111/j.1365-2621.2005.tb09987.x

[25] Andersson, M., Thankachan, P., Muthayya, S., Goud, R., Kurpad, A., Hurrell, R. and Zimmermann, M. (2008) Dual Fortification of Salt with Iodine and Iron: A Randomized, Doubleblind, Controlled Trial of Micronized Ferric Pyrophosphate and Encapsulated Ferrous Fumarate in Southern India. American Journal of Clinical Nutrition, 88, 13781387.

[26] Ballot, D., MacPhail, A., Bothwell, T., Gillooly, M. and Mayet, F. (1989) Fortification of Curry Powder with NaFe(III)EDTA: Report of a Controlled Iron Fortification Trial. American Journal of Clinical Nutrition, 49, 162-169.

[27] Chen, J., Zhao, X., Zhang, X., Yin, S., Piao, J., Huo, J., Yu, B., Qu, N. and Lu, Q. (2007) Studies on the Effectiveness of NaFeEDTA-Fortified Soy Sauce in Controlling Iron Deficiency: A Population-Based Intervention Trial. Food and Nutrition Bulletin, 26, 177-189.

[28] Huo, J., Yang, X., Piao, J., Gao, J., Miao, H., Yu, B., Lu, C. and Chen, J. (2007) NaFeEDTA Fortified Soy Sauce Showed Higher Iron Absorption Rate in Chinese Females. Biomedical and Environmental Sciences, 20, 126-130.

[29] Fidler, M., Davidsson, L., Walczyk, T. and Hurrell, R. (2003) Iron Absorption from Fish Sauce and Soy Sauce Fortified with Sodium Iron EDTA. American Journal of Clinical Nutrition, 78, 274-278.

[30] Van Thuy, P., Berger, J., Davidsson, L., Khan, N., Nga, T., Lam, N., Mai, T., Flowers, C., Nakanishi, Y., Cook, J., Hurrell, R. and Khoi, H. (2003) Regular Consumption of NaFeEDTA Fortified Fish Sauce Improves Iron Status of Anemic Vietnamese Women. American Journal of Clinical Nutrition, 78, 284-290.

[31] Van Thuy, P., Berger, J., Nakanishi, Y., Khan, N., Lynch, S. and Dixon, P. (2005) The Use of NaFeEDTA-Fortified Fish Sauce Is an Effective Tool for Controlling Iron Deficiency in Women of Childbearing Age in Rural Vietnam. Journal of Nutrition, 135, 2596-2601.

[32] Viteri, F., Alvarez, E., Batres, R., Torun, B., Pineda, O., Mejia, L. and Sylui, J. (1995) Fortification of Sugar with Iron Sodium Ethylenediaminotretacetate Improves Iron Status in Semirural Guatemalan Populations. American Journal of Clinical Nutrition, 61, 1153-1163.

[33] Zimmermann, M., Wegmueller, R., Zeder, C., Chaouki, N., Rohner, F., Saissi, M., Torresani, T. and Hurrell, R. (2004) Dual Fortification of Salt with Iodine and Micronized Ferric Pyrophosphate: A Randomized, Double-Blind, Controlled Trial. American Journal of Clinical Nutrition, 80, 952-959.

[34] Uauy, R., Hertrampf, E. and Reddy, M. (2002) Iron Fortification of Foods: Overcoming Technical and Practical Barriers. Journal of Nutrition, 132, 849S-852S.

[35] Jimenez, C., Leets, I., Puche, R., Anzola, E., Montilla, R., Parra, C., Aguilera, A. and Garcia-Casal, M.N. (2010) A Single Dose of Vitamin A Improves Haemoglobin Concentration, Retinol Status and Phagocytic Function of Neutrophils in Preschool Children. British Journal of Nutrition, 103, 798-802. http://dx.doi.org/10.1017/S0007114509992765

[36] Munoz, E., Rosado, J., López, P., Furr, H. and Allen, L (2000) Iron and Zinc Supplementation Improves Indicators of Vitamin A Status of Mexican Preschoolers. American Journal of Clinical Nutrition, 71, 789-794.

[37] Oliveira, J., Michelazzo, F., Stefanello, J. and Rondó, P. (2008) Influence of Iron on Vitamin A Nutritional Status. Nutrition Reviews, 63, 141-147. http://dx.doi.org/10.1111/j.1753-4887.2008.00018.x

[38] Zimmermann, M. (2002) Iron Status Influences the Efficacy of Iodine Prophylaxis in Goitrous Children in Cote d'Ivoire. International Journal of Vitamins and Nutrition Research, 72, 19-25. http://dx.doi.org/10.1024/0300-9831.72.1.19

[39] Garcia-Casal, M.N. (2006) Carotenoids Increase Iron Absorption from Cereal-Based Food in the Human. Nutrition Research, 26, 340-344.

[40] Mei, Z., Cogswell, M., Parvanta, I., Lynch, S., Beard, J., Stolzfus, R. and Gruummer-Strawn L. (2005) Hemoglobin and Ferritin Are Currently the Most Efficient Indicators of Population Response to Iron Interventions: An Analysis of Nine Randomized Controlled Trials. Journal of Nutrition, 135, 1974-1980.

[41] World Health Organization/Centers for Disease Control and Prevention (2007) Assessing the Iron Status of Populations: Including Literature Reviews. Report of a Joint World Health Organization/Centers for Disease Control and Prevention Technical Consultation on the Assessment of Iron Status at the Population Level, Geneva, Switzerland, 6-8 
April 2004, 2nd Edition.

[42] Centers for Disease Control and Prevention, Pan American Health Organization/World Health Organization/U.S. Agency for International Development and The USAID Micronutrient and Child Blindness Project (2008) Monitoring and Evaluating Food Fortification Programs: General Overview. Technical Consultation 7 July 2006. USAID Eds., Washington DC.

[43] Pena-Rosas, J., Parvanta, I., van der Haar, F. and Chapel, T. (2008) Monitoring and Evaluation in Flour Fortification Programs: Design and Implementation Considerations. Nutrition Reviews, 66, 148-162. http://dx.doi.org/10.1111/j.1753-4887.2008.00019.x 